\title{
Zero-Phonon-Line Emission of Single Molecules for Applications in Quantum Information Processing
}

\author{
A. Kiraz ${ }^{a}$, M. Ehrl $^{b}$, Ö. E. Müstecaplığglu ${ }^{a}$, T. Hellerer ${ }^{a}$, C. Bräuchle ${ }^{a}$, and A. Zumbusch ${ }^{b, c}$ \\ ${ }^{a}$ Department of Physics, Koç University, Rumelifeneri Yolu, 34450 Sariyer, Istanbul, Turkey \\ ${ }^{b}$ Department Chemie und Biochemie and Center for Nanoscience, Ludwig-Maximilians \\ Universität München, Butenandtstr. 11, 81377 München, Germany \\ ${ }^{c}$ Department of Physics and Astronomy, University College London, Gower Street, London \\ WC1E 6BT, UK
}

\begin{abstract}
A single photon source which generates transform limited single photons is highly desirable for applications in quantum optics. Transform limited emission guarantees the indistinguishability of the emitted single photons. This, in turn brings groundbreaking applications in linear optics quantum information processing within an experimental reach. Recently, self-assembled InAs quantum dots and trapped atoms have successfully been demonstrated as such sources for highly indistinguishable single photons.

Here, we demonstrate that nearly transform limited zero-phonon-line (ZPL) emission from single molecules can be obtained by using vibronic excitation. Furthermore we report the results of coincidence detection experiments at the output of a Michelson-type interferometer. These experiments reveal Hong-Ou-Mandel correlations as a proof of the indistinguishability of the single photons emitted consecutively from a single molecule. Therefore, single molecules constitute an attractive alternative to single InAs quantum dots and trapped atoms for applications in linear optics quantum information processing.

Experiments were performed with a home-built confocal microscope keeping the sample in a superfluid liquid Helium bath at $1.4 \mathrm{~K}$. We investigated terrylenediimide (TDI) molecules highly diluted in hexadecane (Shpol'skii matrix). A continuous wave single mode dye laser was used for excitation of vibronic transitions of individual molecules. From the integral fluorescence, the ZPL of single molecules was selected with a spectrally narrow interference filter. The ZPL emission was then sent to a scanning Fabry-Perot interferometer for linewidth measurements or a Michelson-type interferometer for coincidence detection.
\end{abstract}

Keywords: Single molecule spectroscopy, quantum optics, quantum information processing, single photon source, photon indistinguishability, two-photon interference, linear optics quantum information processing, vibronic excitation

\section{INTRODUCTION}

Low temperature experiments using single molecules have mostly been performed using the scheme of zerophonon-line (ZPL) excitation. In such studies, the purely electronic ZPL transition of single molecules was excited using a single mode laser while the emission from the Stokes shifted vibronic transitions were collected. This allowed for the observation of almost lifetime limited absorption bands for a selection of dye molecules located in highly ordered host matrices. ${ }^{1,2}$ Two major disadvantages have however accompanied this excitation scheme. Firstly, while ultranorrow absorption bands allowed for a high spectral selectivity, they also yield the scheme vulnerable against spectral jumps. In the event of a spectral jump, the single mode laser emission (only a few $\mathrm{MHz}$ in spectral linewidth) had to be scanned in order to locate the new spectral position of the ZPL absorption. However such a search became practically impossible when the spectral trajectory of single molecules included relatively large spectral jumps $(>30 \mathrm{GHz})$. This, in turn, drastically limited the available host-guest systems that could be investigated. Hence, this high resolution spectroscopic technique could not be applied to many interesting systems such as dye doped polymers or chromophore containing proteins. Secondly, due

Further author information: A.K.: E-mail: akiraz@ku.edu.tr, Telephone: +90 (0) 2123381701 
(a)

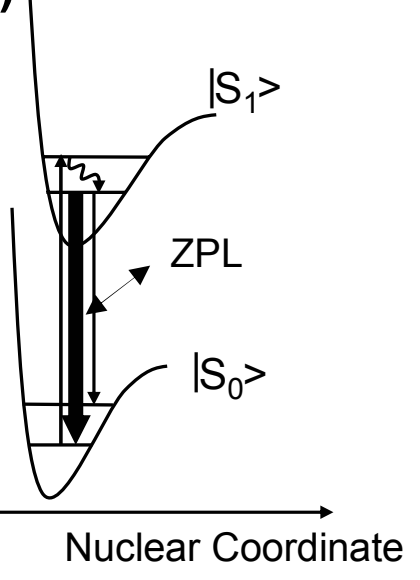

(b)

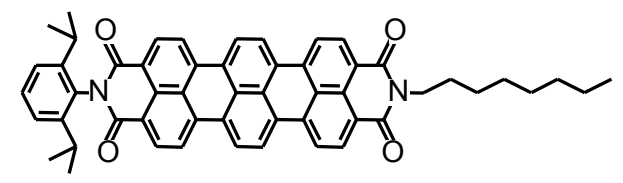

(c)

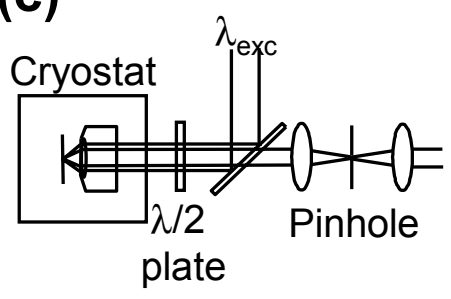

Figure 1. (a) Vibronic excitation scheme. (b) Terrylenediimide (TDI). (c) Low temperature confocal microsocope.

to the large amount of scattering, the ZPL excitation scheme did not allow for the investigation of the ZPL emission from single dye molecules. This prevented the application of this potential source of highly coherent single photons in quantum optics. In this proceeding we describe the technique of vibronic excitation which was shown to solve the two aforementioned disadvantages of the ZPL excitation scheme. ${ }^{3,4}$ We particularly focus on the experiments on quantum optics using the ZPL emission from single molecules.

\section{VIBRONIC EXCITATION}

The vibronic excitation technique relies on the combination of confocal low temperature microscopy with vibronic sideband excitation and spectrally resolved ZPL detection (Fig. 1(a)). At cryogenic temperatures, due to their short lifetimes $(\sim 1-10 \mathrm{ps})$, the vibrational bands of single dye molecules are generally very broad. By contrast, the width of the purely electronic ZPL is typically caused by the long radiative lifetimes ( $\sim$ several ns) of the excited electronic states. The usable absorption width is further increased to tens of $\mathrm{cm}^{-1}$ in systems with a strong electron-phonon coupling due to intense phonon sidebands. Hence, when a vibrational state is excited and ZPL emission is detected in a spectrally resolved manner, spectral jumps of the molecular absorption which remain within the width of the absorption band can be followed. ZPL detection is advantageous as it offers both, high sensitivity and high spectral selectivity since a large part of the excitation energy is emitted in the spectrally narrow ZPL.

This proceeding discusses experiments performed on terrylenediimide (TDI) molecules ${ }^{5}$ (Fig. 1(b)) highly diluted in hexadecane (HD) matrix. Hexadecane forms a Shpol'skii matrix which provides an ordered, partly crystalline surrounding for the TDI molecules. The samples were prepared by adding hexadecane to a solution of TDI in $\mathrm{CHCl}_{3} . \mathrm{CHCl}_{3}$ and $\mathrm{O}_{2}$ were removed by several freeze and thaw cycles using liquid $\mathrm{N}_{2}$ and intermediate evacuation steps. After this, the TDI/hexadecane solution was saturated with Ar. A drop of this solution was then quickly inserted into the precooled cryostat. Finally, the sample was cooled down to $1.4 \mathrm{~K}$ in a liquid He bath. As depicted in Fig. 1(c) in the home-built low temperature confocal microscope, the beam from a single mode dye laser (Coherent, 899-29) is reflected off a dichroic mirror, passed through a $\lambda / 2$ plate before being focused onto the sample with an aspherical lens $(\mathrm{NA}=0.55)$, which is also used for collecting the fluorescence. A galvo optic scanner and a telecentric system, or a a piezo scanner (Attocube ANP100) was used to select single molecules from different spatial positions. After transmision through the $\lambda / 2$ plate and the dichroic mirror, the collected fluorescence was focused onto a pinhole (75 $\mu \mathrm{m}$ or $200 \mu \mathrm{m}$ diameter) and dispersed with a $46 \mathrm{~cm}$ monochromator (Jobin-Yvon, HR460) before detection with a charge coupled device detector (CCD, Acton Research, 128HB). A spectral resolution of $30 \mathrm{GHz}$ was obtained in the spectra taken with the monochromator and the CCD. Alternatively, the signal was sent to a Fabry-Perot spectrum analyzer (Coherent, 

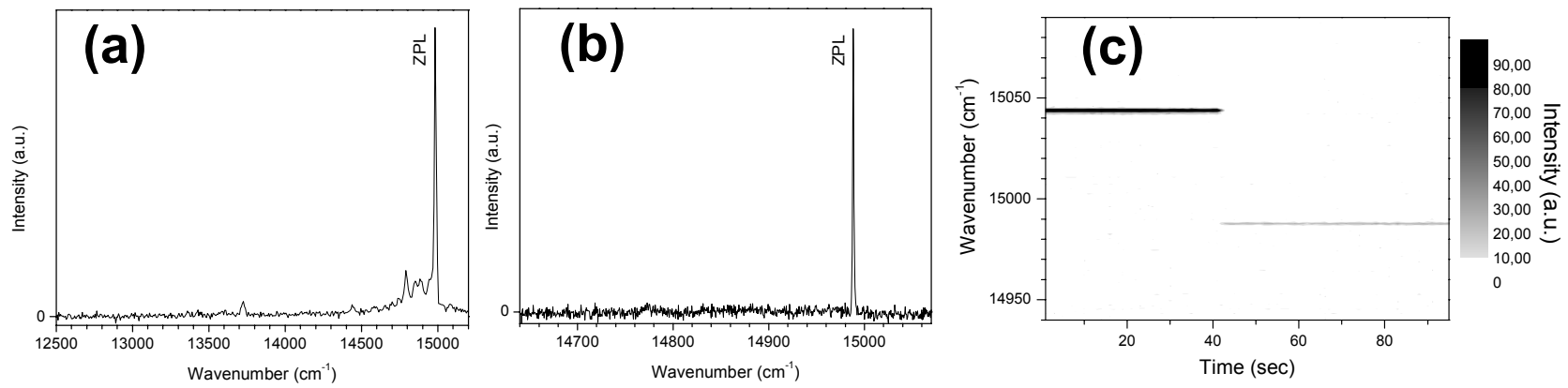

Figure 2. Low (a) and high (b) resolution emission spectra of the TDI molecule taken with under an excitation intensity of $0.5 \mathrm{~mW}$. (c) Consecutive high resolution emission spectra under an excitation intensity of $1.3 \mathrm{~mW}$. Excitation wavelength is $605 \mathrm{~nm}$ in a-c.

240) for linewidth measurements and a home-built Michelson interferometer for experiments on two-photon interference. These parts of the experimental setup are described in the following sections.

Vibronic excitation was previously shown to be applicable to a wide range of systems including amorphous systems. Spectral jumps as large as $80 \mathrm{~cm}^{-1}$ have been demonstrated using this technique. ${ }^{3}$ Figures 2(a) and 2(b) depict a low and high resolution spectrum of the emission from a single TDI molecule respectively, obtained using vibronic excitation. The resolution limited ZPL emission as well as emission due to other vibronic transitions and phonon sidebands are visible in these plots. Under continuous illumination, rare spectral jumps have been observed in the position of the ZPL emission of this molecule as shown in Fig. 2(c). Under $1.3 \mathrm{~mW}$ excitation at $605 \mathrm{~nm}$, the ZPL was initially in the spectral position of $15044 \mathrm{~cm}^{-1}$. After 42 seconds at this position, a jump of $56 \mathrm{~cm}^{-1}$ was observed in the spectral position of the ZPL. Then, the ZPL emission remained in the second spectral position at $14988 \mathrm{~cm}^{-1}$ which was the most stable spectral position for this molecule.

\section{LINEWIDTH MEASUREMENTS}

A scanning Fabry-Perot spectrum analyzer (SA) was employed in order to measure the emission linewidth of the ZPL of single TDI molecules (Fig. 3(a)). The SA had a free spectral range of $1.5 \mathrm{GHz}$. Its finesse and peak transmission were measured to be $\sim 100$ (spectral resolution $\sim 15 \mathrm{MHz}$ ), and $\sim 5 \%$, respectively. A broadband filter (Chroma, HQ 655/60), a color filter (Schott, RG630), and a narrowband interference filter (Omega, 677 $\mathrm{SC} 2, \mathrm{FWHM}=2 \mathrm{~nm}$, angle tunable between $650-677 \mathrm{~nm}$ ) were used in spectrally selecting the ZPL emission. A gated counter (Stanford Instruments, SR400) was used for both external triggering of the SA and accumulation of the avalanche photodiode (APD) counts.

High resolution spectra are obtained by directing this ZPL emission to the SA. A slow scan of the SA reveals spectra of the type depicted in Fig. 3(b). This scan consists of 600 bins, each of which corresponds to different mirror separations of the Fabry-Perot SA. At each bin, the APD counts are integrated for $500 \mathrm{~ms}$. The excitation intensity is $35 \mu \mathrm{W}$, which is much lower than the saturation power. For this reason, no power broadening is expected. The Lorentzian modulations seen in the figure are separated by $1.5 \mathrm{GHz}$, which equals the free-spectral range of the SA. A fit to the data yields a $65 \pm 10 \mathrm{MHz}$ linewidth for the ZPL emission. This corresponds to a coherence decay time of $4.9 \pm 0.9 \mathrm{~ns}$. By comparison, conventional fluorescence excitation spectroscopy of single TDI molecules in hexadecane yield a homogeneous linewidth of $\sim 40 \mathrm{MHz} .{ }^{6}$ Therefore, our measured emission linewidth is within a factor of 1.6 of the transform limit.

Two experimental observations give conclusive evidence that the emission shown in Fig. 2(a) stems from a single molecule. First of all, at the end of this experiment, we increased the excitation power to $10 \mathrm{~mW}$ and were able to thermally activate local degrees of freedom by laser induced heating. This resulted in blinking and a digital spectral jump in the fluorescence spectra (data not shown). Secondly, given the large inhomogeneous broadening of the TDI band in hexadecane, it is highly improbable that several molecules would appear at the same spectral position in the SA. 

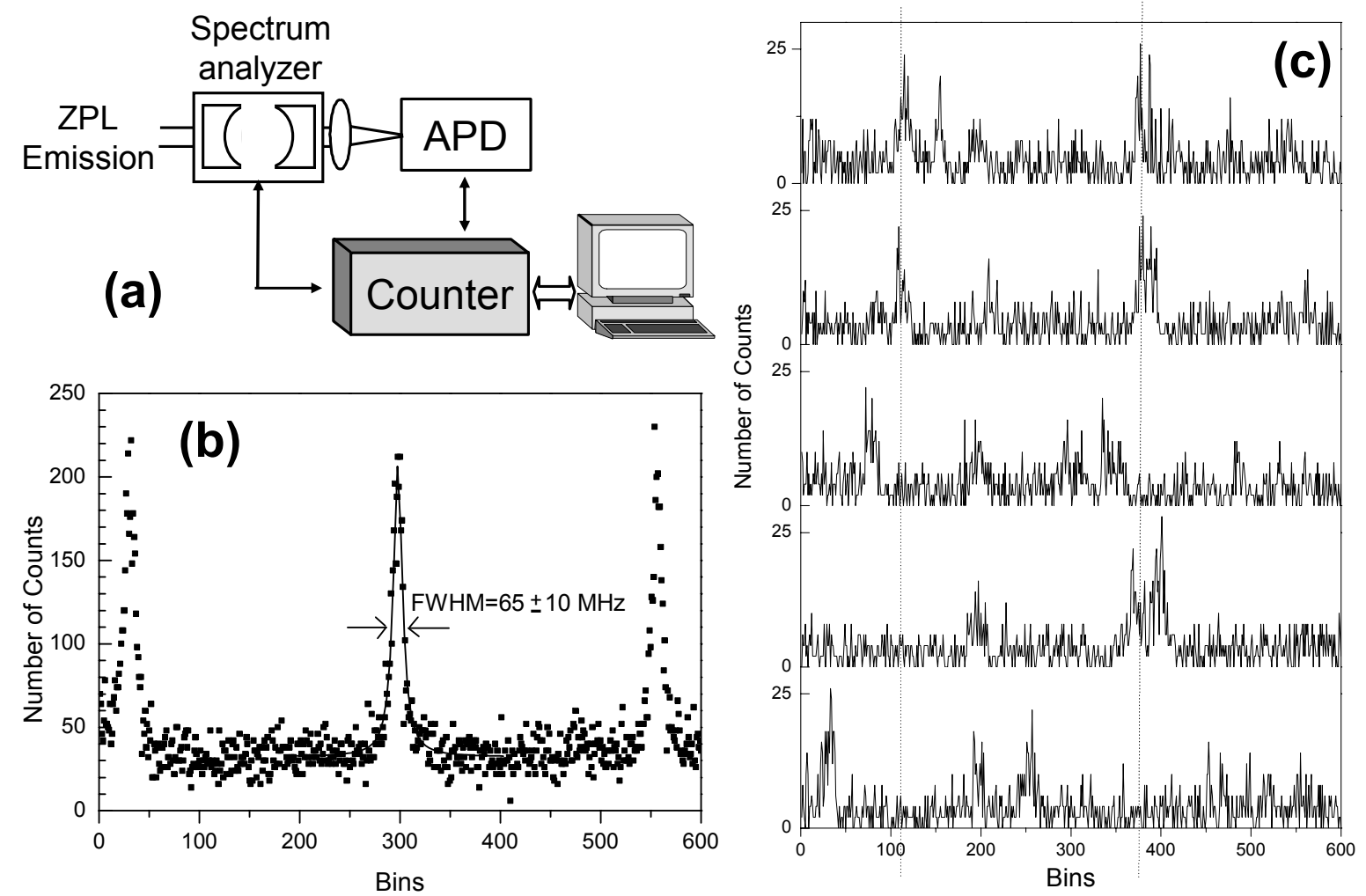

Figure 3. (a) Experimental setup showing the Fabry-Perot spectrum analyzer used for linewidth measurements. (b) Scan of the spectrum analyzer with $500 \mathrm{~ms}$ integration time per bin (separated by $2 \mathrm{~ms}$ dark periods). Excitation at $605 \mathrm{~nm}$ with an intensity of $35 \mu \mathrm{W}$. (c) Consecutive scans of the spectrum analyzer for a single molecule's purely electronic zero-phonon line emission with a $10 \mathrm{~ms}$ integration time per bin (separated by $2 \mathrm{~ms}$ dark periods). Excitation at $608 \mathrm{~nm}$ with an intensity of $500 \mu \mathrm{W}$. Dotted lines are repeated at an interval of $1.5 \mathrm{GHz}$.

We have observed Lorentzian ZPLs with narrow spectra for 8 other molecules. Some of these exhibited spectral dynamics even at moderate excitation intensities. This is shown in Fig. 3, where we plot four fast consecutive scans taken with the ZPL fluorescence from such a molecule, at an excitation intensity of $500 \mu \mathrm{W}$. At each bin, an integration time of $10 \mathrm{~ms}$ was used. From the spectra it is evident that the molecule preferentially occupies several spectral positions. Among those, at least two can be identified as indicated by the straight and dotted lines in the figure. It is however important to note that even molecules which exhibit such spectral dynamics can be suitable sources of indistinguishable single photons. The only requirement which needs to be fulfilled then is that they are spectrally stable on the time scale of the spontaneous emission lifetime. In our case, the spectral jumps take place on a ms time scale whereas the fluorescence lifetime is $10^{6}$ times shorter.

\section{TWO-PHOTON INTERFERENCE}

Two photons which are sent to two different input channels of a 50/50 beam-splitter will interfere depending on their indistinguishability. Ideally indistinguishable photons will exhibit bunching, i.e. they will leave the beam splitter through the same output channel. ${ }^{7}$ Up to date, this two-photon interference phenomenon constituted a major demonstration of the quantum theory of light. In addition, photon-photon interactions mediated by two-photon interference have proven to be very suitable for applications in quantum information science. It is conceivable to perform efficient quantum computation using only indistinguishable single photons, linear optical elements and ideal photon counters. ${ }^{8}$

The availability of true single photon sources is a major practical requirement of the linear optics quantum computation scheme. True single photon sources should provide indistinguishable single photons on demand. 
Photons generated in a parametric down conversion process have been frequently used as such sources. ${ }^{9}$ However, in this source, the number of generated photon pairs is governed by Poissonian statistics. Thus, experiments using parametric down conversion are bound to be performed at low intensities.

Single photon sources based on single two-level emitters promise to overcome this inherent limitation. ${ }^{10-12}$ These single photon sources can emit single photons with a sub-Poissonian photon statistics provided a large collection efficiency. Only very recently, the coherence properties of the emitted single photons have been investigated. Impressive demonstrations of indistinguishability have been given for photons emitted by single quantum dots ${ }^{13}$ and single trapped atoms. ${ }^{14}$ Single molecules are an attractive alternative to both single quantum dots and single trapped atoms. Among the available systems, only single molecules can provide large photon coherence lengths together with large observation times. We have readily observed photon coherence lengths close to $4.9 \mathrm{~ns}^{4}$ for stable single molecules which can fluoresce over 20 days without photobleaching at cryogenic temperatures. ${ }^{15}$ In the following, we report on Hong-Ou-Mandel experiments using the zerophonon-line (ZPL) emission from a single terrylenediimide (TDI) molecule. As a result of these experiments, we demonstrate the two-photon interference phenomenon.

The Michelson interferometer used in our experiment is depicted in Figures 4(a) and 4(b). In contrast to the recently reported two-photon interference experiments using single quantum dots ${ }^{13}$ and single trapped atoms, ${ }^{14}$ we have employed continuous wave (cw) excitation in our experiment. Under cw excitation, a path length difference which is larger than half of the coherence length of the emitted photons is selected. This ensures that two independent photons will simultaneously arrive at two inputs of the beam splitter with a certain probability. The signature of two-photon interference is then revealed as the lack of coincidence counts at the output of the beam splitter.

As depicted in Fig. 4(a), we have implemented a rotatable $\lambda / 2$ plate in the longer arm of the interferometer in order to achieve parallel or orthogonal polarizations in channels 1 and 2. Considering parallel polarizations, in the limit of large $\Delta t(\Delta t>1 / \gamma)$ and small $\tau(\tau<\Delta t)$, a coincidence counting experiment between channels 3 and 4 reveals the normalized second-order coherence function ${ }^{16}$ :

$$
g_{34 \|}^{(2)}(\tau)=\frac{1}{2}\left(g^{(2)}(\tau)+1\right)-\frac{\sin ^{2} \theta \cos ^{2} \theta}{\cos ^{4} \theta+\sin ^{4} \theta}\left|g^{(1)}(\tau)\right|^{2} .
$$

$\gamma=\Gamma_{\text {spon }} / 2+\gamma_{\text {pure }}$ is the total dephasing rate of the ZPL including spontaneous emission $\left(\Gamma_{\text {spon }}\right)$ and dephasing due to other sources $\left(\gamma_{\text {pure }}\right)$. In this equation $g^{(1)}(\tau), g^{(2)}(\tau)$ correspond to the normalized first-order and secondorder coherence functions of the ZPL emission of a single molecule respectively. Transmission and reflection in the beam-splitter are noted as $\cos ^{2} \theta$ and $\sin ^{2} \theta$ respectively.

For mutually orthogonal polarizations, photons at both input channels are completely distinguishable, and no effect of two-photon interference should be observed. For this case, the result of a coincidence experiment between channels 3 and 4 , reveals:

$$
g_{34 \perp}^{(2)}(\tau)=\frac{1}{2}\left(g^{(2)}(\tau)+1\right) .
$$

Examplary solutions of $g_{34}^{(2)}(\tau)$ for both mutually parallel (solid) and orthogonal (dashed) polarizations are depicted in Fig. 4(c). In the solid curve, the signature of two-photon interference is the absence of coincidence events around zero delay time.

For the two-photon interference experiments, the confocal microscope was equipped with a piezo scanner in order to select single molecules from different spatial positions in the sample. After being filtered with a narrow band interference filter $(\mathrm{FWHM}=1 \mathrm{~nm}$, angle tunable between $645-670 \mathrm{~nm})$, ZPL fluorescence from a single TDI molecule was directed into the homebuilt Michelson interferometer. The path length difference between the two arms of the interferometer was equivalent to a temporal delay of $\Delta t=4.6 \mathrm{~ns}$. One of its arms was equipped with a rotatable $\lambda / 2$-plate. The output signals at the beam splitter were detected using APDs in a Hanbury-Brown and Twiss configuration. The APD outputs were sent to a time to amplitude converter (TAC) as start and stop pulses. After adding an electronic delay on the stop pulse, the output of the TAC which was stored in a 
(a)
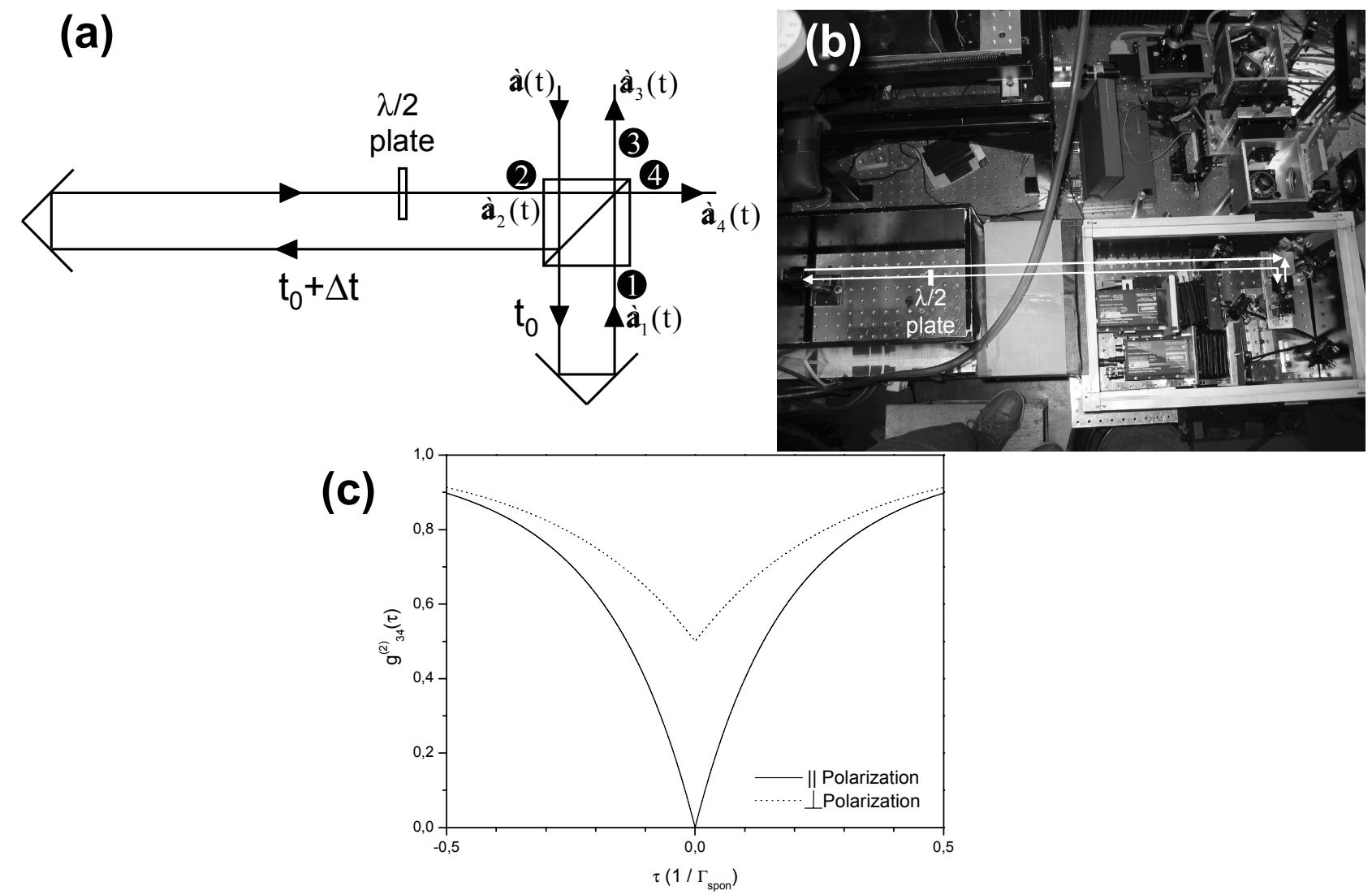

Figure 4. (a)Michelson interferometer. (b) A view of the experimental setup showing the Michelson interferometer. (c) Solution of $g_{34}^{(2)}(\tau)$ for large $\Delta t(\Delta t \gg 1 / \gamma)$ and small $\tau$ considering parallel and ortohogonal polarizations. Parameter values are $W_{P}=0.25 \Gamma_{\text {spon }}, \gamma_{\text {dep }}=0.3 \Gamma_{\text {spon }}$, and $\theta=\pi / 4$. $W_{P}$ is the effective pumping rate of the two-level emitter.

multi channel analyzer (MCA) revealed the second order correlation function $\left(g_{34}^{(2)}(\tau)\right)$. A temporal resolution of 420 ps was achieved.

Fig. 5(a) depicts the results of coincidence measurements for the case when the $\lambda / 2$-plate introduced no polarization change in the long arm of the interferometer (parallel polarizations). At the zero delay position, the value of $g^{(2)}(0)$ becomes 0.4 . Fig. 5(b) depicts the result of the coincidence detection experiment when we have rotated the polarization of the fluorescence in the long interferometer arm by $90^{\circ}$. Clearly, the resulting correlation curve exhibits higher $g^{(2)}$ values around zero delay time. The normalized difference between the two curves is shown in Fig. 5(c). In this curve, the dip around zero delay time is above the noise level, and constitutes the proof for the observed Hong-Ou-Mandel correlations. As a comparison, Figures 5(d) and 5(e) show the normalized difference between the data of Fig. 5(a) and another measurement with parallel polarizations, as well as for the data of Fig. 5(b) and another measurement with orthogonal polarization on the same molecule. In contrast to Fig. 5(c), no signal above the noise level is seen at zero delay in both of these cases.

The observed $g^{(2)}(0)=0.4$ for parallel polarizations corresponds to a photon indistinguishability of at least $\sim 0.2$ instead of the ideal value of 1 . Two reasons can be responsible for this relatively low level of indistinguishability. Firstly, imperfections in the mode matching of our interferometer are a source of contrast reduction. Because of its higher throughput, a conventional Michelson interferometer was preferred over a setup using a single mode glass fibre in our experiment. Secondly, spectral jumps of the molecule could deteriorate the contrast. If these jumps occur on the time scale of the excited state lifetime, they will give rise to dephasing and a corresponding line broadening. While we did not determine the ZPL emission linewidth of this molecule, a fit to the correlation measurement in Fig. 5 (a) reveals $\Gamma_{\text {spon }} \sim 1 /(3 n s)$ and $\gamma_{\text {pure }} \sim 1 /(4 n s)$, hence a photon 

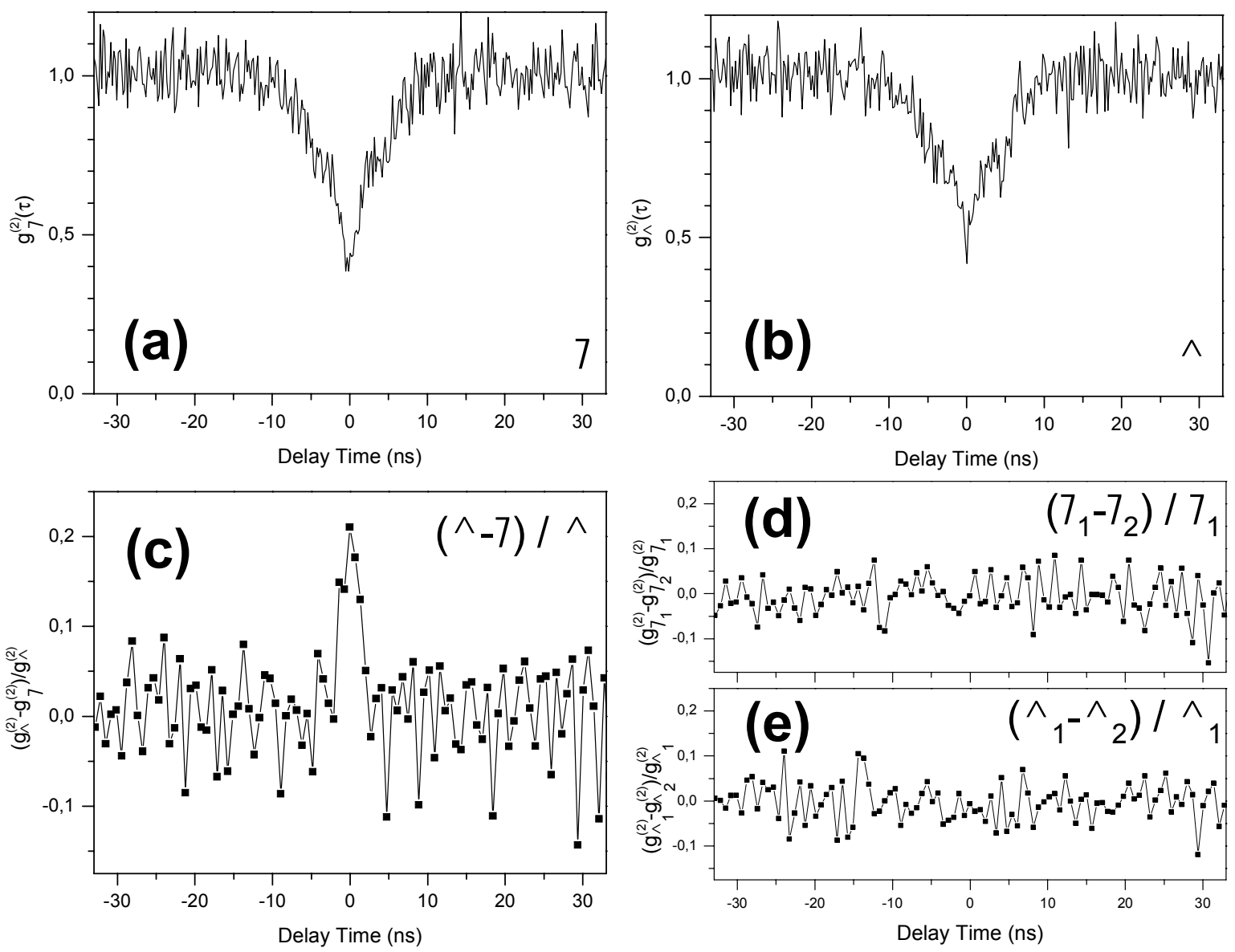

Figure 5. Measured normalized second-order photon correlation functions for parallel (a) and orthogonal (b) polarizations. (c) Difference between the correlation functions $g_{\perp}^{(2)}$ and $g_{\|}^{(2)}$ normalized to $g_{\perp}^{(2)}$. Normalized difference between two measurements at parallel (d) and orthogonal (e) polarizations. Excitation is at $605 \mathrm{~nm}$ with an intensity of $1 \mathrm{~mW}$ in a-e. Binning of the data is 2 in a and b, and 7 in c-e.

coherence time of $\sim 2.5 \mathrm{~ns}$.

\section{CONCLUSIONS AND OUTLOOK}

In conclusion, we have used the newly developed technique of vibronic excitation at cryogenic temperatures in order to analyze the coherence properties of the zero-phonon line (ZPL) emission of single molecules. Our firstorder coherence measurement experiments revealed nearly transform limited ZPL emission from a single TDI molecule in hexadecane with a coherence decay time of 4.9 ns. We used a high resolution Fabry-Perot spectrum analyzer in these experiments.

Using this highly coherent source of single photons, we have performed two-photon interference experiments under continuous wave excitation. In these experiments using a Michelson interferometer, a photon indistinguishability of at least $\sim 0.2$ was observed as compared to the ideal value of 1 .

Our results demonstrate that the ZPL emission of single molecules can be used as a source of indistinguishable single photons. This yields single molecules as an attractive alternative to single InAs quantum dots and trapped atoms for applications in linear optics quantum information processing. The two-photon interference experiments presented in this proceeding can easily be extended to include a pulsed excitation scheme. Although pulsed excitation scheme is not necessary for the demonstration of two-photon interference, it will be important for 
practical applications. Our approach offers new possibilities for performing linear optics quantum information experiments like those reported in ${ }^{13,17,18}$ using larger coherence times.

\section{ACKNOWLEDGMENTS}

The authors thank K. Müllen for a gift of TDI and A. Imamoḡlu for helpful discussions. This work was supported by the Deutsche Forschungsgemeinschaft, SFB533, and the Alexander von Humboldt Foundation (A.K.). O.E.M. acknowledges support from TÜBA/GEBIP award. Partial support was provided by European Commission (EC) Grant No: IST-511616 PHOREMOST.

\section{REFERENCES}

1. P. Tamarat, A. Maali, B. Lounis, and M. Orrit J. Phys. Chem. A 104, p. 1, 2000.

2. W. E. Moerner J. Phys. Chem. B 106, p. 910, 2002.

3. A. Kiraz, M. Ehrl, C. Bräuchle, and A. Zumbusch J. Chem. Phys. 118, pp. 10821-10824, 2003.

4. A. Kiraz, M. Ehrl, C. Bräuchle, and A. Zumbusch Appl. Phys. Lett 85, p. 920, 2004.

5. S. Mais, J. Tittel, T. Basché, C. Bräuchle, W. Göhde, H. Fuchs, G. Müller, and K. Müllen J. Phys. Chem. 101, p. 8435, 1997.

6. S. Mais, T. Basché, G. Müller, K. Müllen, and C. Bräuchle Chem. Phys. 247, pp. 41-52, 1999.

7. P. Grangier Nature 419, p. 577, 2002.

8. E. Knill, R. Laflamme, and G. J. Milburn Nature (London) 409, p. 46, 2001.

9. C. K. Hong, Z. Y. Ou, and L. Mandel Phys. Rev. Lett. 59, pp. 2044-2046, 1987.

10. C. Brunel, B. Lounis, P. Tamarat, and M. Orrit Phys. Rev. Lett. 83, p. 2722, 1999.

11. P. Michler, A. Kiraz, C. Becher, W. V. Schoenfeld, P. M. Petroff, L. Zhang, E. Hu, and A. Imamoḡlu Science 290, p. 2282, 2000.

12. A. Kuhn, M. Hennrich, and G. Rempe, "Deterministic single-photon source for distributed quantum networking," Phys. Rev. Lett. 89, p. 067901, 2002.

13. C. Santori, D. Fattal, J. Vuckovic, G. S. Solomon, and Y. Yamamoto Nature 419, pp. 594-597, 2002.

14. T. Legero, T. Wilk, M. Hennrich, G. Rempe, and A. Kuhn Phys. Rev. Lett. 93, p. 070503, 2004.

15. F. Kulzer, S. Kummer, R. Matzke, C. Bräuchle, and T. Basché Nature 387, p. 688, 1997.

16. A. Kiraz, M. Atatüre, and A. Imamoḡlu Phys. Rev. A 69, p. 032305, 2004.

17. D. Fattal, K. Inoue, J. Vuckovic, C. Santori, G. S. Solomon, and Y. Yamamoto Phys. Rev. Lett. 92, p. 037903, 2004.

18. D. Fattal, E. Diamanti, K. Inoue, and Y. Yamamoto Phys. Rev. Lett. 92, p. 037904, 2004. 